\title{
Safety of same-day administration of gemcitabine plus cisplatin chemotherapy for urothelial carcinoma
}

\author{
IBUKI TSURU, FUSAKO NIIMI, SACHI HONDA and TAKESHI AZUMA \\ Department of Urology, Tokyo Metropolitan Tama Medical Center, Fuchu, Tokyo 183-0042, Japan
}

Received July 3, 2020; Accepted December 18, 2020

DOI: $10.3892 / \mathrm{mco} .2021 .2219$

\begin{abstract}
The present study aimed to examine the safety of a gemcitabine and cisplatin (GC) combination chemotherapy regimen with short hydration for the treatment of urothelial cancer administered on the same day (same day regimen). Patients with locally advanced or metastatic urothelial cancer received the same-day GC regimen with short hydration every 4 weeks, and their serum creatinine $(\mathrm{Cr})$ level was measured to assess renal function using linear mixed model analysis. A total of 20 patients receiving the same-day regimen exhibited no significant change in their serum $\mathrm{Cr}$ level; nor was there any significant change in the serum $\mathrm{Cr}$ level between patients who received the same day regimen and those who received the drugs on different days. The present study demonstrated that the same-day regimen was safe for patients with urothelial cancer.
\end{abstract}

\section{Introduction}

Two platinum-based regimens are considered to be the standard treatments for UC. One of these consists of a combination of methotrexate, vinblastine, doxorubicin, and cisplatin (MVAC) (1). The other consists of a combination of gemcitabine and cisplatin (GC) (2). A phase III clinical trial of the regimens demonstrated similar efficacy in terms of the overall survival and response rates. However, GC had a better safety profile. In addition, Robinson demonstrated a superior cost-utility profile for GC (3). Based on these data, GC is now widely used.

Our previous report demonstrated that outpatient GC chemotherapy with short hydration was safe for patients with UC and contributed to improving their quality of life (QOL) (4). The study examined different-day administration of gemcitabine

Correspondence to: Dr Takeshi Azuma, Department of Urology, Tokyo Metropolitan Tama Medical Center, 2-9-2 Musashidai, Fuchu, Tokyo 183-0042, Japan

E-mail: tazuma-tky@umin.ac.jp

Key words: short hydration, gemcitabine, cisplatin, urothelial carcinoma and cisplatin. Moreover, a same-day GC regimen with short hydration for lung cancer (5) and bile duct cancer (6) demonstrated its potential to improve patients' QOL by decreasing the required number of regular hospital visits.

In the present study, the safety of the same-day GC regimen with short hydration in patients with UC was assessed prospectively with the aim of further improving patients' QOL.

\section{Patients and methods}

Study design and patients. The present, single-center, phase-1 study (UMIN000031015) enrolled patients aged 20 years or older with locally advanced or metastatic UC who were eligible for GC chemotherapy based on their physician's judgement and had an Eastern Cooperative Oncology Group performance status of 2 or less. The patients were screened for adequate hematological and end-organ function before enrollment. A historical control comprising 61 patients who previously received gemcitabine and cisplatin on different days (the different-day regimen) with short hydration at the Tokyo Metropolitan Tama Medical Center between 2010 and 2014 were also enrolled.

Safety evaluation was performed according to protocol. The severity of adverse events was graded using the National Cancer Institute Common Terminology Criteria for Adverse Events (version 5.0) (7).

The present study was conducted in accordance with the Good Clinical Practice guidelines and conforms to the Declaration of Helsinki. All the patients provided written informed consent. The protocol (28-83) was approved by the institutional review board at Tokyo Metropolitan Tama Medical Center on January 10, 2017.

Treatment. Table I shows the same-day GC chemotherapy regimen with short hydration. The patients received gemcitabine $1,000 \mathrm{mg} / \mathrm{m}^{2}$ on days 1,8 , and 15 plus cisplatin $56-70 \mathrm{mg} / \mathrm{m}^{2}$ on day 1 . The cisplatin dosage was determined by the following criteria: $\mathrm{Ccr}>60 \mathrm{ml} / \mathrm{min}, 100 \%$ dose; $40<\mathrm{Ccr}<60 \mathrm{ml} / \mathrm{min}, 80 \%$ dose. Cisplatin was administered with adequate pre- and post-treatment hydration, and the cycle was repeated every 28 days. As an anti-emetic, dexamethasone $9.9 \mathrm{mg}$ and palonosetron $0.75 \mathrm{mg}$ were administered intravenously, and aprepitant $125 \mathrm{mg}$ was administered orally on day 2. Dexamethasone $8 \mathrm{mg}$ and aprepitant $80 \mathrm{mg}$ were 
administered orally on days 3-6 and days 3-4, respectively. Supportive care, including administration of analgesics, blood transfusions, and antibiotics, was performed as required. Granulocyte colony stimulating factor was not used routinely. Same-day GC chemotherapy was first performed with short hydration at our inpatient center. The patients chose outpatient or inpatient chemotherapy after the second round of chemotherapy.

To monitor renal function, the serum creatinine $(\mathrm{Cr})$ level was measured, and the estimated glomerular filtration rate (eGFR) was calculated on days 3, 8, 15, 22 and 29. Changes in the serum $\mathrm{Cr}$ level of patients receiving short hydration were compared with those of the consecutive hydration group using a linear mixed model. Further, to evaluate long-term renal function, the serum $\mathrm{Cr}$ level and eGFR before, and at one year after, chemotherapy in the surviving patients were assessed [eGFR $=194 \times$ (serum creatinine) $-1.094 \times$ age $-0.287 \times(0.739$ if female)].

Statistical analysis. Categorical variables were compared using unpaired t-test, Pearson's chi-square test or Fisher's exact test. The paired t-test was used to assess changes in renal function. All statistical analyses were carried out with SPSS version 21 (IBM Corp.) and $\mathrm{P}<0.05$ was considered to indicate statistical significance.

\section{Results}

Patients. Twenty patients received the same-day GC chemotherapy regimen with short hydration between January 2017 and March 2018 on an outpatient basis. Table II shows the patient characteristics. The average age was 69.2 years (range, 53 to 77 years), and the sex distribution was 18 men and two women. Nine patients underwent a nephrectomy, and two patients had a single kidney due to hydronephrosis caused by a malignancy. The median serum $\mathrm{Cr}$ value and eGFR value was $0.97 \mathrm{mg} / \mathrm{dl}$ (range 0.55-1.35) and $59.5 \mathrm{ml} / \mathrm{min}$ (range, 44-87), respectively. There were 11 and seven patients with an eGFR value $<60 \mathrm{ml} / \mathrm{min}$ and $<50 \mathrm{ml} / \mathrm{min}$, respectively. The median follow-up was 18.2 months.

Treatment. In total, 86 courses of the same-day GC chemotherapy regimen with short hydration were performed on an outpatient basis. The average number of chemotherapy courses was 4.3 (range, 2-10). Of all the patients receiving short hydration, nine $(45 \%)$ received cisplatin $70 \mathrm{mg} / \mathrm{m}^{2}$ while $11(55 \%)$ received $56 \mathrm{mg} / \mathrm{m}^{2}$ with short hydration at the outpatient chemotherapy center.

Adverse effects. Three patients had grade 3 neutropenia, one patient had grade 4 thrombocytopenia, and no patient had anemia (Table III). Only one patient presented grade 1 nausea. None of the patients experienced dehydration. Two patients had grade 1 elevation of liver enzymes (Table III). All produced more than $3,000 \mathrm{ml} /$ day of urine during days 2-7. Three patients experienced Grade 1 acute kidney injury according to the National Cancer Institute Common Terminology Criteria for Adverse Events (NCI CTCAE) version 5.0. In all the cases, renal toxicity was completely reversible with oral hydration alone.
Renal function. Fig. 1 shows the changes in serum $\mathrm{Cr}$ level and eGFR in each patient receiving the same-day GC chemotherapy regimen with short hydration. There was no significant difference in the serum $\mathrm{Cr}$ level or eGFR between the period prior to, and one month after, treatment $(\mathrm{P}=0.495$ and 0.691 , respectively). Fig. 2 shows the changes in the serum $\mathrm{Cr}$ level in each patient receiving the GC same day regimen with short hydration. The historical control comprised patients who had received gemcitabine and cisplatin with short hydration on different days. Table II shows their characteristics. No significant difference was found between the characteristics of the groups. Our previous study demonstrated no change in the serum $\mathrm{Cr}$ level in the historical control. The change in the serum $\mathrm{Cr}$ level in the same-day regimen group, further examined using linear mixed model analysis, demonstrated no significant change $(\mathrm{P}=0.513)$. Next, the serum $\mathrm{Cr}$ level in each group around the time of the chemotherapy was analyzed using linear mixed model analysis and revealed no significant difference between the different-day and same-day regimen groups $(\mathrm{P}=0.262)$.

Next, to evaluate the long-term effect of short hydration, 20 patients who were alive one year after the short hydration chemotherapy (Fig. 3) were assessed. Four patients had received adjuvant chemotherapy. There was no significant difference in the serum $\mathrm{Cr}$ level or eGFR between the period prior to, and one year after, the administration of the same-day GC chemotherapy regimen with short hydration $(\mathrm{P}=0.810$ and 0.610 , respectively).

\section{Discussion}

Our previous study demonstrated the safety of outpatient GC chemotherapy with short hydration and its potential to improve patients' quality of life (QOL) (4). To improve patients' QOL further, the safety and effectiveness of same-day GC regimen with short hydration in UC patients were assessed prospectively.

The same-day GC chemotherapy with short hydration is the standard treatment for metastatic lung cancer (5) or bile duct cancer (6) while the different-day regimen is the standard treatment for UC (2). Patients with UC are usually older and have a solitary functional kidney due to a nephrectomy or hydronephrosis $(8,9)$. These factors can sometimes induce severe adverse responses to treatment. In the present cohort, four patients were older than 75 years and 11 patients had a solitary functional kidney. The serum $\mathrm{Cr}$ values never worsened after the same-day GC chemotherapy with short hydration. Patients with the different-day chemotherapy with short hydration were enrolled as the historical control, but no significant difference between the groups was found.

The frequency and the severity of adverse events were similar between the groups. Chemotherapy-induced nausea and vomiting, which can reduce oral intake and sometimes induce dehydration, were observed in patients in both treatment groups. In the current study, none of the patients with short hydration experienced vomiting thanks to the use of anti-emetics (serotonin and neurokinin 1 receptor antagonists) (10) although some patients experienced mild nausea. Decrease in appetite was denied. There were also no severe 
Table I. Regimen.

Drugs

Fluids

Timing, $\min$

Day 1

Gemcitabine

Potassium chloride (10 mEq) + Magnesium sulfate (8 mEq)

Dexamethasone $(9.9 \mathrm{mg})+$ Palonosetron $(0.75 \mathrm{mg})$

Cisplatin

Potassium chloride $10 \mathrm{mEq}$

Day 8,15

Gemcitabine
$100 \mathrm{ml} 0.9 \% \mathrm{NaCl}$ solution $\quad 30$

$500 \mathrm{ml} 0.45 \% \mathrm{NaCl}$ solution $\quad 60$

$500 \mathrm{ml} 0.45 \% \mathrm{NaCl}$ solution $\quad 60$

$50 \mathrm{ml} 0.9 \% \mathrm{NaCl}$ solution $\quad 30$

$200 \mathrm{ml} \mathrm{20 \%} \mathrm{Mannitol} \quad 30$

$300 \mathrm{ml} 0.9 \% \mathrm{NaCl}$ solution $\quad 60$

$500 \mathrm{ml} 0.45 \% \mathrm{NaCl}$ solution $\quad 60$

$500 \mathrm{ml} 0.45 \% \mathrm{NaCl}$ solution $\quad 60$

$100 \mathrm{ml} 0.9 \% \mathrm{NaCl}$ solution

Dexamethasone $8 \mathrm{mg}$ was administered on days 2-5. Aprepitant $80 \mathrm{mg}$ was administered orally on days 1-3.
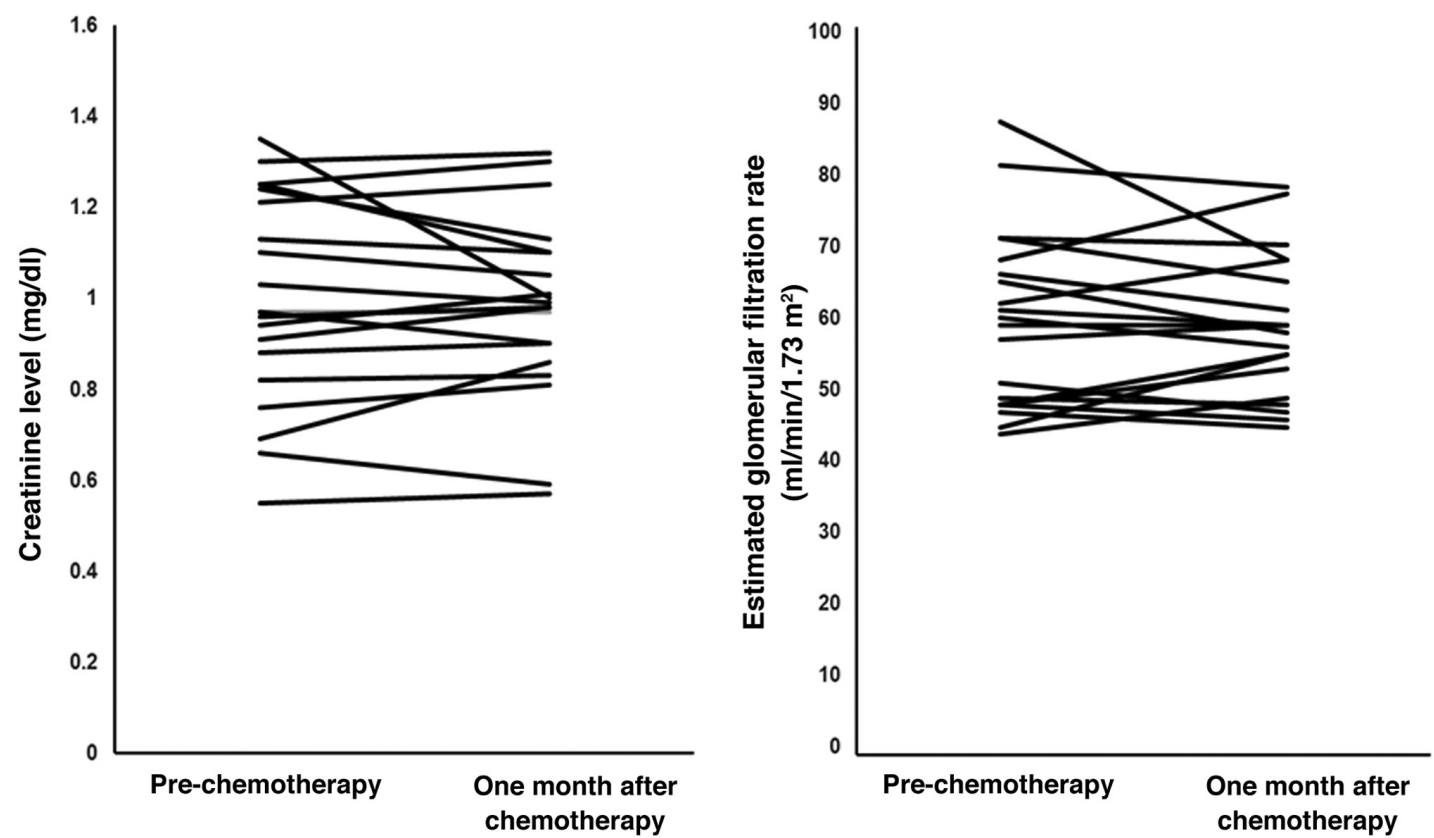

Figure 1. Difference in serum creatinine level and glomerular filtration rate before and 1 month after same-day gemcitabine and cisplatin regimen with short hydration.
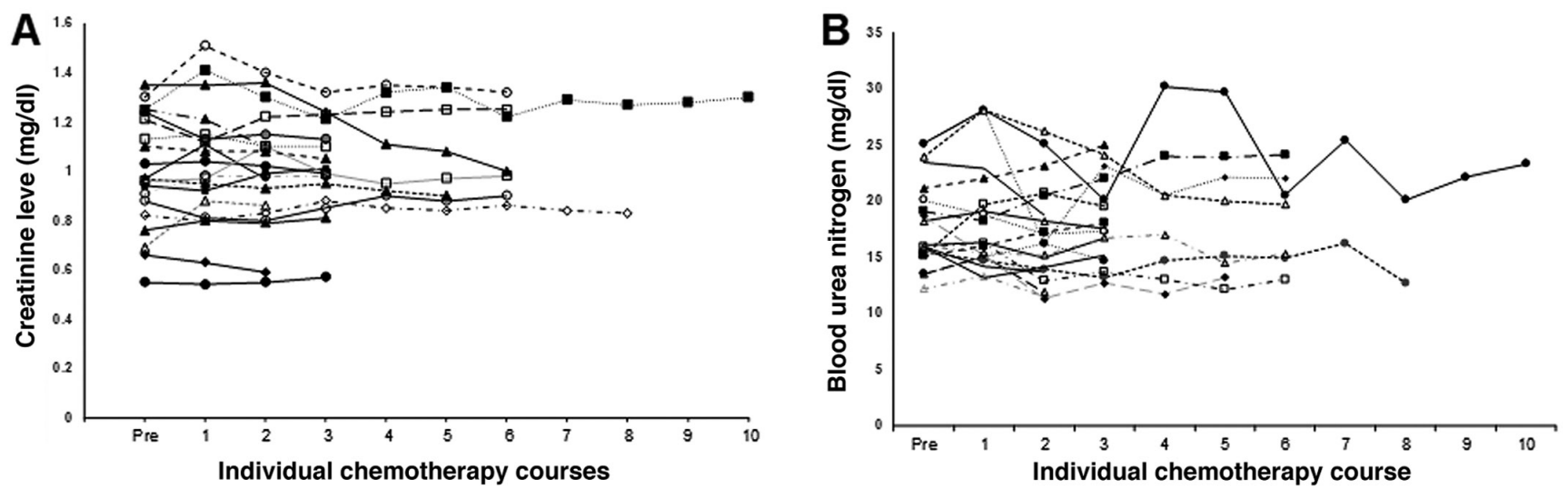

Figure 2. Time course of (A) serum creatinine level and (B) blood urea nitrogen in each patient receiving same-day gemcitabine and cisplatin regimen with short hydration. 
Table II. Patient characteristics.

\begin{tabular}{|c|c|c|c|}
\hline Characteristics & Same day & Different day & P-value \\
\hline \multicolumn{4}{|l|}{ Age, years } \\
\hline Average & 69.2 years & 71.3 years & 0.72 \\
\hline Range & $53-77$ years & $40-86$ years & \\
\hline Sex, n $(\%)$ & & 0.80 & \\
\hline Male & $18(90)$ & $56(92)$ & \\
\hline Female & $2(10)$ & $5(8)$ & \\
\hline Eastern Cooperative Oncology Group performance status, n (\%) & & & 0.48 \\
\hline 0 & 20 & 57 & \\
\hline 1 & 0 & 3 & \\
\hline 2 & 0 & 1 & \\
\hline 3 & 0 & 0 & \\
\hline 4 & 0 & 0 & \\
\hline Disease, n (\%) & & & 0.51 \\
\hline Bladder cancer & $11(55)$ & $26(42)$ & \\
\hline Upper urinary cancer & $9(45)$ & $33(54)$ & \\
\hline Others & $0(0)$ & $2(4)$ & \\
\hline Nephrectomy, n (\%) & & & 0.75 \\
\hline Yes & $9(45)$ & $25(41)$ & \\
\hline No & $11(55)$ & $36(59)$ & \\
\hline Hydronephrosis, n (\%) & & & 0.20 \\
\hline Yes & $2(18)$ & $14(22)$ & \\
\hline No & $9(37)$ & $22(37)$ & \\
\hline Serum Creatinine (mg/dl) before chemotherapy, n (\%) & & & 0.65 \\
\hline$\leq 1.0$ & $11(45)$ & $30(49)$ & \\
\hline$>1.0$ & $9(55)$ & $31(51)$ & \\
\hline Estimated glomerular filtration rate ( $\mathrm{ml} / \mathrm{min})$ before chemotherapy, $\mathrm{n}(\%)$ & & & 0.84 \\
\hline$\geq 60$ & $9(45)$ & $29(48)$ & \\
\hline$<60$ & $11(55)$ & $32(52)$ & \\
\hline
\end{tabular}

Unpaired t-test was used to evaluate age. Pearson's $\chi^{2}$ test was used for the other parameters.
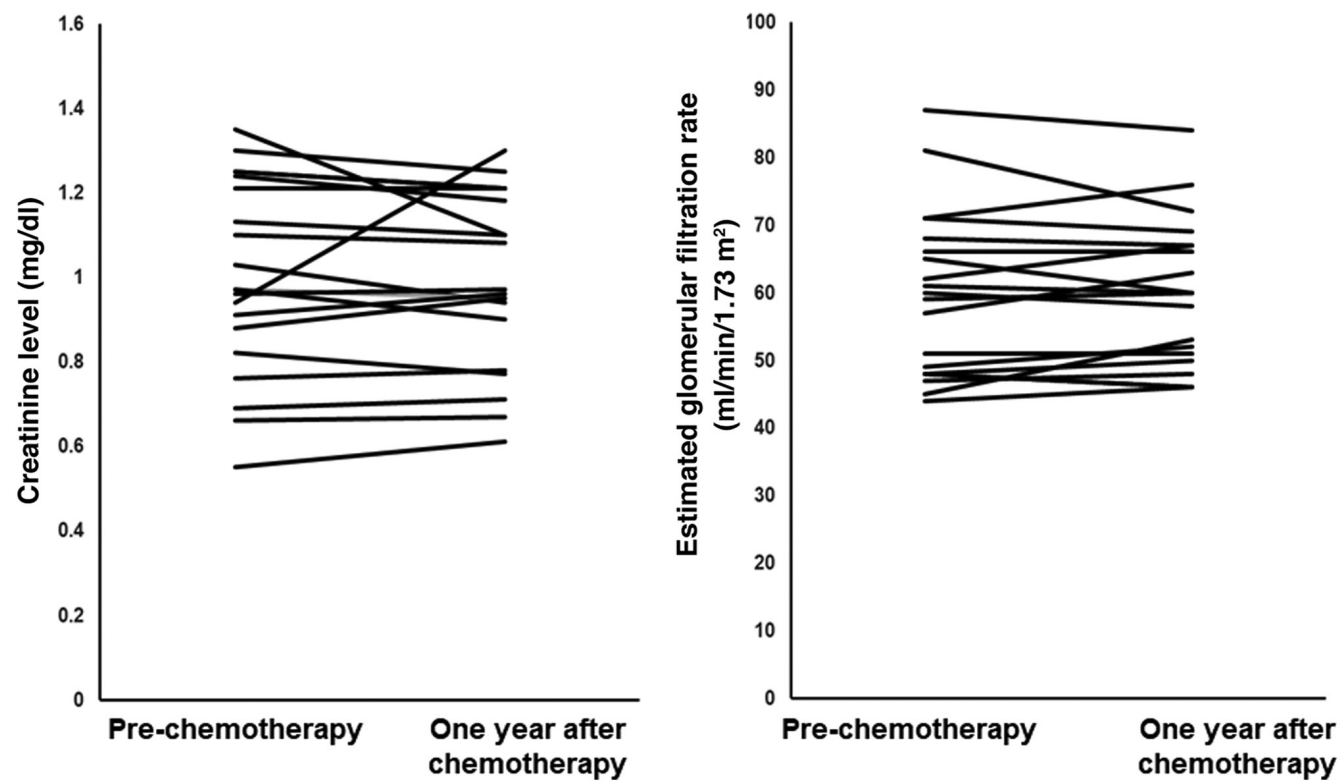

Figure 3. Differences in serum creatinine level and glomerular filtration rate before and 1 year after same-day gemcitabine and cisplatin regimen with short hydration 
Table III. Safety summary.

\begin{tabular}{|c|c|c|c|c|c|c|c|c|}
\hline \multirow[b]{2}{*}{ CTCAE version 5.0} & \multicolumn{2}{|c|}{ Grade 1 to 2} & \multicolumn{2}{|c|}{ Grade 3} & \multicolumn{2}{|c|}{ Grade 4} & \multicolumn{2}{|c|}{ Total } \\
\hline & No. & $\%$ & No. & $\%$ & No. & $\%$ & No. & $\%$ \\
\hline \multicolumn{9}{|l|}{ Non-hematologic } \\
\hline Fatigue & 1 & 5 & 0 & 0 & 0 & 0 & 1 & 5 \\
\hline Nausea & 1 & 5 & 0 & 0 & 0 & 0 & 1 & 5 \\
\hline \multicolumn{9}{|l|}{ Hematologic } \\
\hline Leukopenia & 6 & 30 & 3 & 15 & 0 & 0 & 9 & 45 \\
\hline Thrombocytopenia & 3 & 15 & 0 & 0 & 1 & 5 & 4 & 20 \\
\hline Neutropenia & 6 & 30 & 3 & 15 & 0 & 0 & 9 & 45 \\
\hline \multicolumn{9}{|l|}{ Hepatic } \\
\hline Increase in alanine aminotransferase & 2 & 10 & 0 & 0 & 0 & 0 & 0 & 0 \\
\hline Increase in aspartate aminotransferase & 2 & 10 & 0 & 0 & 0 & 0 & 0 & 0 \\
\hline
\end{tabular}

CTCAE, National Cancer Institute Common Terminology Criteria for Adverse Events.

hematological toxicities in patients receiving the same-day GC chemotherapy regimen with short hydration. Three patients had grade 3 neutropenia, and one patient had grade 4 thrombopenia, but none of the patients had febrile neutropenia or required a blood transfusion.

Naiki et al (11) demonstrated the safety of the same-day GC regimen with short hydration in patients with UC. The present study differs from the latter study in two respects. First, because the present study was based on actual clinical situations, the regimen was administered on an outpatient basis. In our previous study, we demonstrated that the different-day GC chemotherapy with short hydration could be performed safely on an outpatient basis and improve patients' QOL. The same-day GC regimen with short hydration was able further to improve patients' QOL. Second, the present study $(70 \%)$ included more patients with solitary kidney than a previous study $(30 \%)$, enabling renal function to be analyzed in greater detail. We used the linear mixed model, which is superior to repeated measures ANOVA for analyzing data collected multiple times. Our data showed that there was no significant change in the slope of the incline for the time course of the serum creatinine level. In addition, renal function was analyzed one year after chemotherapy because of reports of late nephrotoxicity induced by cisplatin.

In this study, QOL data were not collected because the control data were unable to be established. However, the new regimen described herein demonstrated the ability to decrease the number of regular hospital visits and improve patients' QOL. Further, the decrease in hospital visits may also help to reduce medical expenses and conserve medical resources.

In the present study, six partial responses $(38 \%)$, three stable diseases (19\%), and seven progressive diseases (43\%) according to the Response Evaluation Criteria in Solid Tumors (RECIST), version 1.1. were observed, in line with the findings of previous reports. However, the small sample size limited our assessment of the effect of the same-day GC chemotherapy regimen.
The present study demonstrated that the same-day GC chemotherapy regimen with short hydration was safe for older patients with urological cancer with compromised renal function as well as for outpatients with UC.

\section{Acknowledgements}

Not applicable.

\section{Funding}

No funding was received.

\section{Availability of data and materials}

The datasets used and/or analyzed during the current study are available from the corresponding author on reasonable request.

\section{Authors' contributions}

TA designed the present study and critically revised the manuscript. IT, FN and SH performed data collection and analyzed the data. TA wrote the manuscript. All authors read and approved the final manuscript.

\section{Ethics approval and consent to participate}

The protocol (28-83) was approved by the Institutional Review Board at Tokyo Metropolitan Tama Medical Center. All patients provided written informed consent.

\section{Patient consent for publication}

Not applicable.

\section{Competing interests}

The authors declare that they have no competing interests. 


\section{References}

1. Sternberg CN, Yagoda A, Scher HI, Watson RC, Ahmed T, Weiselberg LR, Geller N, Hollander PS, Herr HW, Sogani PC, et al: Preliminary results of M-VAC (methotrexate, vinblastine, doxorubicin and cisplatin) for transitional cell carcinoma of the urothelium. J Urol 133: 403-407, 1985.

2. von der Maase H, Hansen SW, Roberts JT, Dogliotti L, Oliver T, Moore MJ, Bodrogi I, Albers P, Knuth A, Lippert CM, et al: Gemcitabine and cisplatin versus methotrexate, vinblastine, doxorubicin, and cisplatin in advanced or metastatic bladder cancer: Results of a large, randomized, multinational, multicenter, phase III study. J Clin Oncol 18: 3068-3077, 2000.

3. Robinson P, Maase H, Bhalla S, Kielhorn A, Aristides M, Brown A and Tilden D: Cost-utility analysis of the GC versus MVAC regimens for the treatment of locally advanced or metastatic bladder cancer. Expert Rev Pharmacoecon Outcomes Res 4: 27-38, 2004.

4. Azuma T, Matayoshi Y, Sato Y, Sato Y, Nagase Y and Oshi M: The safety and effect of chemotherapy with short hydration for urothelial cancer on patients' quality of life. Jpn J Clin Oncol 46: 958-963, 2016.

5. Tiseo M, Martelli O, Mancuso A, Sormani MP, Bruzzi P, Di Salvia R, De Marinis F and Ardizzoni A: Short hydration regimen and nephrotoxicity of intermediate to high-dose cisplatin-based chemotherapy for outpatient treatment in lung cancer and mesothelioma. Tumori 93: 138-144, 2007.

6. Abdel-Rahman O, Elsayed Z and Elhalawani H: Gemcitabinebased chemotherapy for advanced biliary tract carcinomas. Cochrane Database Syst Rev 4: CD011746, 2018.
7. National Cancer Institute: Common Terminology Criteria for Adverse Events (CTCAE). https://ctep.cancer.gov/protocoldevelopment/electronic applications/ctc.htm. Accessed Sep 12, 2020.

8. Dash A, Galsky MD, Vickers AJ, Serio AM, Koppie TM, Dalbagni $G$ and Bochner BH: Impact of renal impairment on eligibility for adjuvant cisplatin-based chemotherapy in patients with urothelial carcinoma of the bladder. Cancer 107: 506-513, 2006.

9. Kaag MG, O'Malley RL, O'Malley P, Godoy G, Chen M, Smaldone MC, Hrebinko RL, Raman JD, Bochner B, Dalbagni G, et al: Changes in renal function following nephroureterectomy may affect the use of perioperative chemotherapy. Eur Urol 58: 581-587, 2010.

10. Basch E, Prestrud AA, Hesketh PJ, Kris MG, Feyer PC, Somerfield MR, Chesney M, Clark-Snow RA, Flaherty AM, Freundlich B, et al; American Society of Clinical Oncology: Antiemetics: American Society of Clinical Oncology clinical practice guideline update. J Clin Oncol 29: 4189-4198, 2011.

11. Naiki T, Sugiyama Y, Tasaki Y, Iida K, Etani T, Hamamoto S, Nagai T, Nozaki S, Ando R, Kawai N, et al: Efficacy of a newly modified short hydration method for gemcitabine and cisplatin combination chemotherapy in patients with urothelial carcinoma. Oncology 98: 612-620, 2020. 